ARTICLE

Received 11 Apr 2013 | Accepted 8 Jul 2013 | Published 3 Sep 2013 DOl: 10.1038/ncomms3278

\title{
Twin-induced one-dimensional homojunctions yield high quantum efficiency for solar hydrogen generation
}

Maochang Liu ${ }^{1, \star}$, Dengwei Jing ${ }^{1, \star}$, Zhaohui Zhou $^{1} \&$ Liejin Guo ${ }^{1}$

Efficient charge separation is of crucial importance for the improvement of photocatalytic activity for solar hydrogen evolution. Here we report efficient photo-generated charge separation by twin-induced one-dimensional homojunctions with type-Il staggered band alignment, using a ternary chalcogenate, i.e. $\mathrm{Cd}_{0.5} \mathrm{Zn}_{0.5} \mathrm{~S}$ nanorod as a model material. The quantum efficiency of solar hydrogen evolution over this photocatalyst, without noble metal loading, reaches $62 \%$. Unlike traditional heterojunctions, doping or combination of additional elements are not needed for the formation of this junction, which permits us to tune the band structures of semiconductors to the specific application in a more precise way. Our results highlight the power of forming long-range ordered homojunctions at the nanoscale for photocatalytic and photoelectrochemical applications.

\footnotetext{
${ }^{1}$ International Research Center for Renewable Energy, State Key Laboratory of Multiphase Flow in Power Engineering, Xi'an Jiaotong University, Xi'an, Shanxi 710049, China. * These authors contributed equally to this work. Correspondence and requests for materials should be addressed to L.G. (email: lj-guo@mail.xjtu.edu.cn).
} 
s Fujishima and Honda ${ }^{1}$ first presented the concept of photo-electrochemical water splitting into $\mathrm{H}_{2}$ and $\mathrm{O}_{2}$ over a $\mathrm{TiO}_{2}$ electrode, photocatalytic hydrogen production from water by semiconductors has attracted considerable interest ${ }^{1-6}$. To date, a number of semiconductor photocatalysts including sulfides, nitrides, metal/metallic oxides, and others have been investigated ${ }^{1,7-15}$. However, further commercial development, as well as large scale manufacture of photocatalysts, is still restricted by their low activity and high cost.

Photocatalytic reaction over semiconductors is usually considered as the direct absorption of a photon by the energy band gap of materials. Upon photon excitation, the separated electron and hole migrate to the semiconductor surface where photocatalytic reactions occur ${ }^{6}$. Therefore, at the most basic level, enhancing charge separation and preventing their recombination before they move to the surface-reactive sites of the photocatalyst have key roles in determining the solar energy conversion efficiency. To this end, many strategies have been investigated, including in situ photodeposition of noble metal as cocatalysts onto photocatalyst surface, chemical doping to form donor or acceptor energy levels within the band gap of the photocatalyst, and development of Z-scheme systems ${ }^{16-20}$. Among these approaches, coupling of two different materials or phases at the nanoscale to form heterojunctions has proven successful, owing to improved interfacial charge transfer and therefore effective charge separation ${ }^{20-23}$. However, because of the random and disordered distributions of heterojunctions within these materials, it could be speculative to solely attribute the observed difference in photocatalytic activity to the existence of the junctions. On the other hand, doping or combination of additional elements is often needed for the formation of these hetero-junctions, making it difficult to precisely tailor the band structures of semiconductors to the specific application.

Twinning in nanocrystals, especially in one-dimensional (1D) nanomaterials, has been investigated over III-V and II-VI semiconductors anticipating their potential applications in optical and electronic devices ${ }^{24-26}$. Usually, twin planes periodically occur with a constant spacing and have a long-range order, resulting in twinning superlattice (TSL) in a specific dimension ${ }^{24-29}$. Thus, mini-bands could be generated in this crystal structure ${ }^{24}$. Calculation also reveals that unusual states of charge carriers may emerge from the twinning plane as electron wavefunction is discontinuous around this domain ${ }^{30}$. Figure 1 presents typical face-centered cubic lattice symmetry with two coherent twin planes parallel to each other (see Fig. 1a) and the resulting TSL domain in a 1D 'nanorod' as viewed from [ -110$]$ and high-index [ - hhk] directions (see Fig. 1b,c). Although a convincing example is still needed, we assume that the mini-bands formed by TSL could be very useful for charge separation in photocatalysts. Recently, we found that spherical twin-containing $\mathrm{Cd}_{0.5} \mathrm{Zn}_{0.5} \mathrm{~S}$ photocatalyst without loading noble metal exhibited excellent photocatalytic activity for $\mathrm{H}_{2}$ evolution, with a quantum efficiency (QE) of $43 \%$ at $425 \mathrm{~nm}$ (ref. 31). Nevertheless, tangible proof for the contribution of nano-twins to charge separation in photocatalytic materials is still highly desired. Moreover, the low density of twin planes and the poorly controlled crystal shape in these twin-containing $\mathrm{Cd}_{0.5} \mathrm{Zn}_{0.5} \mathrm{~S}$ photocatalyts indicate a large space for the further improvement of its photocatalytic efficiency.

Here we report a twinned $\mathrm{Cd}_{0.5} \mathrm{Zn}_{0.5} \mathrm{~S}$ anisotropic nanocrystal with controllable aspect ratios and a high proportion of long-range ordered twin planes, which give rise to the alternating zinc-blende (ZB) and wurtzite (WZ) segments along $<111>$ direction of the designed material. Further investigations reveal that the type-II staggered band alignment formed between the $\mathrm{ZB}$ and $\mathrm{WZ}$ segments, leads to the generation of myriad homojunctions in a specific dimension. These homojunctions were found to be highly efficient in charge separation and photocatalytic hydrogen production with a remarkable QE of $62 \%$. We believe that our findings could also be extended to other semiconductor materials and provide a new and generalized approach for the development of novel active photocatalytic materials.

\section{Results}

Characterizations of twinned $\mathbf{C d}_{0.5} \mathbf{Z n}_{0.5} \mathrm{~S}$ nanorods. Figure 2 shows typical electron microscopic images, as well as selected area electron diffraction patterns, of the $\mathrm{Cd}_{0.5} \mathrm{Zn}_{0.5} \mathrm{~S}-\mathrm{EN} 10$ nanocrystals. The scanning electron microscopy (SEM) images (Fig. 2a) clearly show the existence of mono-dispersed short nanocrystals in a polygonal cross profile with average diameter of around $60 \mathrm{~nm}$ and aspect ratio of 4 (as statistically estimated in Supplementary Fig. S1). A closer examination (inset of Fig. 2a)
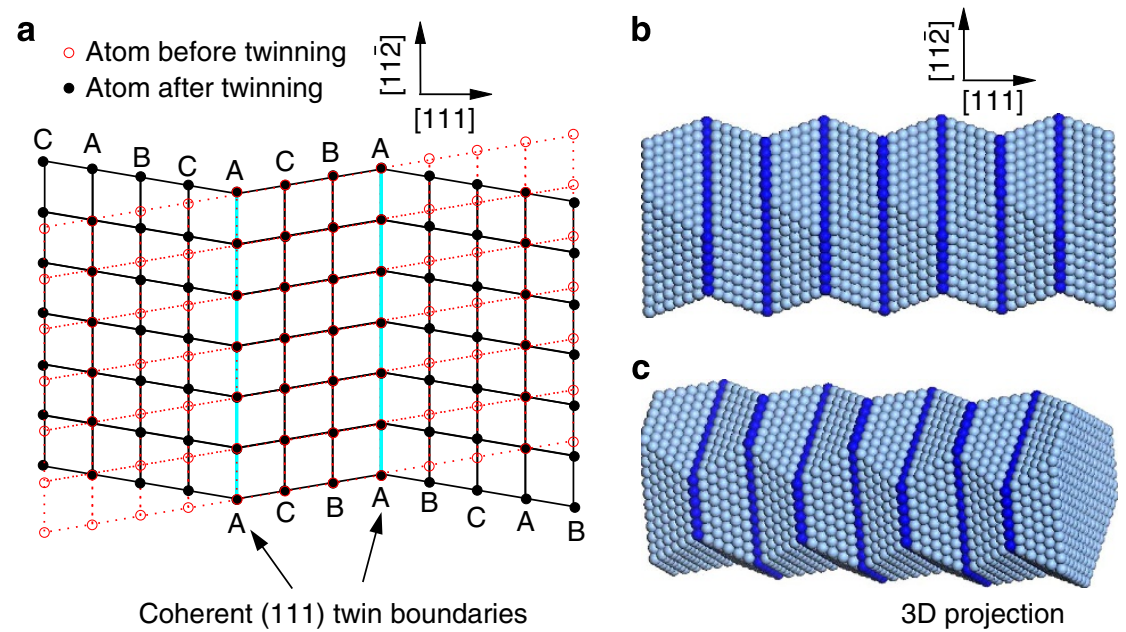

Figure 1 | Structural models of a typical twin nanocrystal. (a) Two (111)/[11-2] type coherent twin planes involved in a zinc blende (ZB) FCC lattice matrix. The thicker cyan lines represent twin planes. Red circles and solid black solid are lattice configurations before and after twining, respectively. Structurally, twin planes within one crystal are usually parallel to each other as they index to the same crystal plane. Besides, lattices on one side are symmetrical with that of the other side of the twin plane (like a mirror). (b) A twinned nanorod as seen from the [ - 110] direction. (c) Three-dimensional projection as observed from a high-index [ - hhk] direction. The dark blue layers in $\mathbf{b}, \mathbf{c}$ indicate the twin planes. 
indicates that the two tips of the nanocrystal were notably different, with the cross-section of one head smaller than that of the main body, and the other gradually tapered to its ends. The anisotropic geometry was also verified by transmission electron microscope (TEM) analysis as shown in Fig. 2b. For simplicity, these anisotropic $\mathrm{Cd}_{0.5} \mathrm{Zn}_{0.5} \mathrm{~S}$ nanocrystals were collectively called 'nanorods'. The TEM images (inset of Fig. 2b) further revealed that the 'nanorods' consisted of a high density of stacking faults with parallel distribution. The high-resolution TEM image (Fig. 2c) showed that most of these parallel dislocations were coherent twin boundaries. The fringes with a lattice spacing of ca. $0.32 \mathrm{~nm}$ could be indexed to the $\{111\}$ planes of the ZB lattice. Further investigation on morphology (Fig. 2c) showed that their surfaces were not as smooth as those found in the SEM images. Instead, the nanorods were composed of non-uniformed zigzag structures originating from the suddenly interrupted lattice continuity by twin boundaries. Most of the zigzag segments had a longitude size (spacing) $<10 \mathrm{~nm}$ due to the high density of twin boundaries (see Supplementary Fig. S2). The nano-twin structure was also supported by the selected area electron diffraction
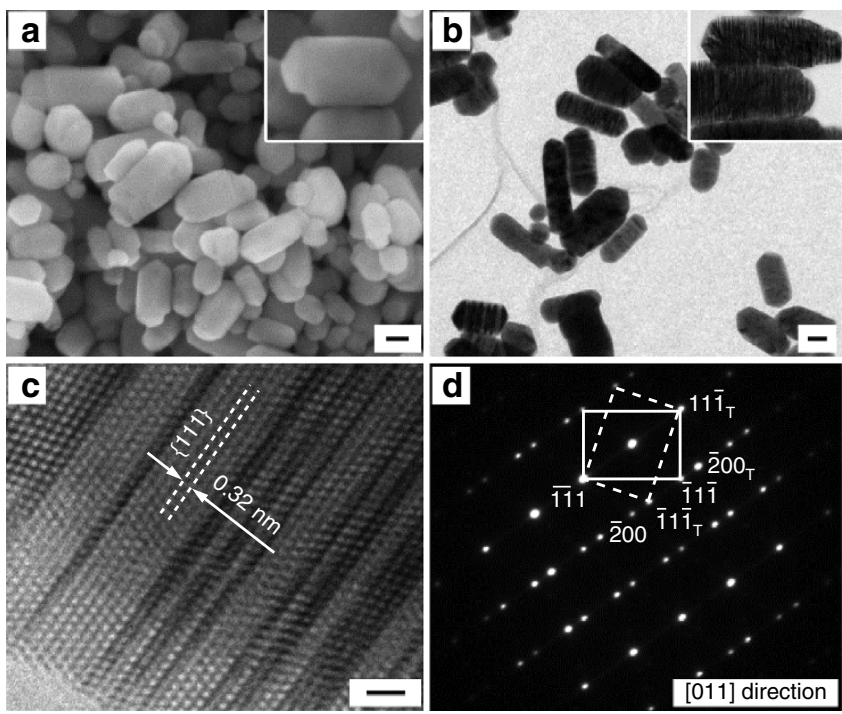

Figure 2 | Morphology and structure of $\mathbf{C d}_{0.5} \mathbf{Z n}_{\mathbf{0 . 5}} \mathbf{S}$ nanorod. $(\mathbf{a}, \mathbf{b}) \mathrm{SEM}$ and TEM images of the as-prepared $\mathrm{Cd}_{0.5} \mathrm{Zn}_{0.5} \mathrm{~S}$ nanorods with high density of twin planes. (c) HRTEM of the nanorod with $\{111\}$ twin plane.

(d) the selected area electron diffraction (SAED) patterns confirming the formation of (111)/[11-2]-type slips in nano-twins. The insets in $\mathbf{a}, \mathbf{b}$ are single particles with higher resolution. Scale bars in (a-c) are $50 \mathrm{~nm}, 50 \mathrm{~nm}$ and $1 \mathrm{~nm}$, respectively. pattern shown in Fig. 2d. Ordered spots appeared at the sites onethird from the normal spots known as the diffraction of coherent twins ${ }^{32,33}$. Schematic illustration of the crystal structure for this twinned $\mathrm{Cd}_{0.5} \mathrm{Zn}_{0.5} \mathrm{~S}$ nanocrystal obtained by using atomic models is presented in Supplementary Fig. S3. Clearly, Cd/Zn layers and S layers symmetrically distribute around the twin plane (either the metal layer or S layer) at the center region of the lattice as shown in Supplementary Fig. S3a. Particularly, the deviated atoms after twinning from the original sites should be responsible for the unique diffraction features.

To confirm the solid solution formation and exclude the possibility of phase segregation between $\mathrm{CdS}$ and $\mathrm{ZnS}$, we have carried out the elemental analysis with scanning TEM (STEM) and energy dispersive X-ray (EDX) analysis. According to the mapping and 'longitude' and 'latitude' line scans over a typical twinning $\mathrm{Cd}_{0.5} \mathrm{Zn}_{0.5} \mathrm{~S}$ nanorod, as shown in Supplementary Fig. S4, Cd, Zn and S are homogeneously distributed in the $\mathrm{Cd}_{0.5} \mathrm{Zn}_{0.5} \mathrm{~S}$ nanorod, respectively. In addition, the successive shifts of powder X-ray diffraction peaks, as well as ultraviolet-vis absorption edges for other $\mathrm{Cd}_{1-x} \mathrm{Zn}_{x} \mathrm{~S}(0 \leq x \leq 1)$ nanocrystals, as shown by Supplementary Fig. S5, provided further proofs of the formation of $\mathrm{Cd}_{0.5} \mathrm{Zn}_{0.5} \mathrm{~S}$ solid solution. Photocatalytic tests over these $\mathrm{Cd}_{1-x} \mathrm{Zn}_{x} \mathrm{~S}$ nanocrystals demonstrated significant improvement in the energy conversion efficiency over $\mathrm{Cd}_{0.5} \mathrm{Zn}_{0.5}$ S-EN10 photocatalyst (Fig. 3a). The initial hydrogen production rate under visible light irradiation reached $2.58 \mathrm{mmol} \mathrm{h}^{-1}$ with a QE of $62 \%$ at $425 \mathrm{~nm}$, as also evidenced by Supplementary Movie 1. To the best of our knowledge, this is one of the highest efficiency achieved over powdered photocatalysts for visible-lightdriven hydrogen production, without employing any cocatalysts. The photocatalyst also have relatively good stability with maintained activity after $25 \mathrm{~h}$ of testing (Fig. 3b). For those $\mathrm{Cd}_{1-x} \mathrm{Zn}_{x} \mathrm{~S}$ with $x<0.5$, owing to their lower conduction band (CB) position, less overpotential are available for hydrogen production. On the other hand, $x$ larger than 0.5 are also not favoured due to reduced visible light absorption. Up to now, higher efficiencies have only been reported over $\mathrm{Pt} / \mathrm{PdS} / \mathrm{CdS}$ and PdS/CdS photocatalysts, However, both of them are noblemetal containing ${ }^{20}$. Based on the $\mathrm{H}_{2}$ generation results in Fig. 3b, we also evaluated the kinetics of the reaction. The rate constant (k) was obtained by plotting $-\ln \left(\mathrm{C}_{\mathrm{t}, \mathrm{Na}_{2} \mathrm{~S}} / \mathrm{C}_{0, \mathrm{Na}_{2} \mathrm{~S}}\right)$ against the reaction time, assuming that the reaction fits the first order kinetics and considering that one mole of $\mathrm{H}_{2}$ generation always corresponds to one mole of $\mathrm{Na}_{2} \mathrm{~S}$ consumption ${ }^{34}$. As shown in Supplementary Fig. S6, the reaction well fitted the first order kinetics with the almost unchanged $k$ values of $0.043 \mathrm{~h}^{-1}$, $0.038 \mathrm{~h}^{-1}, 0.036 \mathrm{~h}^{-1}$ and $0.037 \mathrm{~h}^{-1}$, respectively, for each run. This again demonstrated the stable photocatalytic behavior of our material.
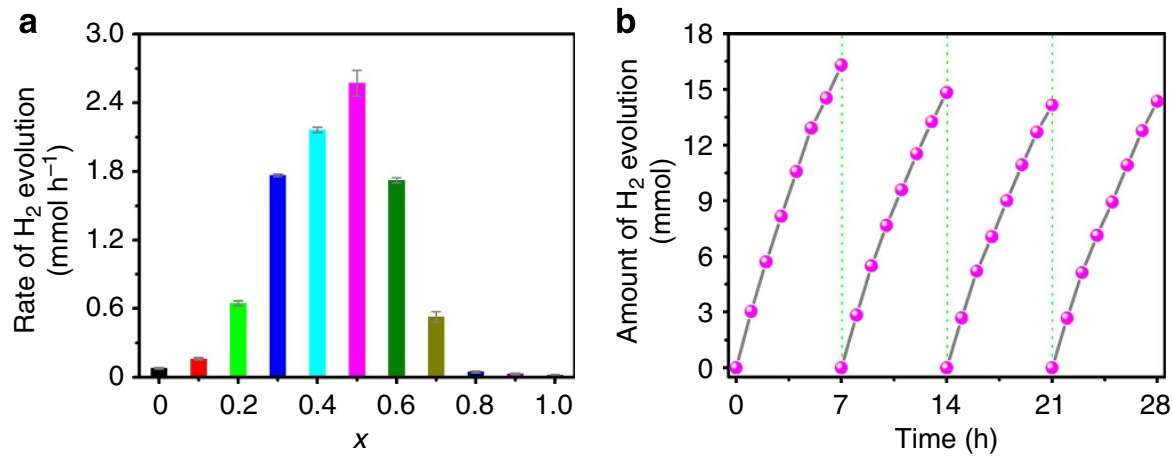

Figure 3 | Photocatalytic performances of $\mathbf{C d}_{\mathbf{1}_{-x}} \mathbf{Z n}_{\mathbf{x}} \mathbf{S}$-EN10. (a) The initial hydrogen generation rate over $\mathrm{Cd}_{1-x} \mathrm{Zn} \mathrm{n}_{\mathbf{x}} \mathrm{S}$ solid solutions of various $\mathrm{Cd} / \mathrm{Zn}$ molar ratios by $5 \mathrm{~h}$ test. (b) Time course for hydrogen generation over $\mathrm{Cd}_{0.5} \mathrm{Zn}_{0.5} \mathrm{~S}$-EN10 nanorods. Error bars in a are s.e. values of three tests $(n=3)$. 
A QE of $43 \%$ over spherical $\mathrm{Cd}_{0.5} \mathrm{Zn}_{0.5} \mathrm{~S}$ photocatalyst possessing twinning domain has been reported ${ }^{31}$. Given the isotropic properties of this spherical $\mathrm{Cd}_{0.5} \mathrm{Zn}_{0.5} \mathrm{~S}$ photocatalyst, the formation of TSL with a long-range order is not possible. However, this may be available in $1 \mathrm{D}$ material such as nanorods and nanowires. As expected, the high activity of present $\mathrm{Cd}_{0.5} \mathrm{Zn}_{0.5} \mathrm{~S}$ nanorods indicates the significant contribution of long-range TSL-induced charge mobility to the enhanced activity of the photocatalyst.

Charge separation over the twin-induced homojunction. To understand the relationship between the twinning nanostructures and their resultant performances, the fundamental lattice symmetry of the twin plane in a nanocrystal must be emphasized. In general, binary compounds with close-packed crystal structures can crystallize into $\mathrm{ZB}, \mathrm{WZ}$, or their mixtures, in which one layer can be defined as a bilayer composed of paired atoms of its components. For instance, in $\mathrm{ZB} \mathrm{ZnS,} \mathrm{the} \mathrm{paired} \mathrm{atoms} \mathrm{are}$ composed of one $\mathrm{Zn}$ atom and one $\mathrm{S}$ atom. The lattice sequences of $\mathrm{ZB}$ and $\mathrm{WZ}$ structures are $\mathrm{AaBbCcAaBbCc}$ and $\mathrm{AaBbAaBb}$, respectively (the paired letters, for example, $\mathrm{Aa}$ or $\mathrm{Bb}$, represents a bilayer). However, the ordered structures can be broken when stacking faults are introduced. As a twin plane in ZB structure in the $\langle 111\rangle$ direction can be considered as a monolayer of WZ structure ${ }^{25}$, it means that a stacking fault in $\mathrm{ZB}$ (WZ) can create a segment of $\mathrm{WZ}(\mathrm{ZB})$. For example, in the $\mathrm{ZB}$ structure, misplacement of a bilayer to yield a sequence of $\mathrm{AaBbCcAaCcBbAc}$ results in a symmetry structure with a mirror plan $\boldsymbol{A a}$, which is known as a twin plane or twin boundary, whereas the consequent $C \boldsymbol{A} A \boldsymbol{a} C \boldsymbol{c}$ segment can be recognized as a three-bilayer WZ structure ${ }^{28,35}$. Therefore, the appearance of these twin boundaries in nanocrystals can suddenly break the continuity of the $\mathrm{ZB}$ lattice constitution and generate a myriad of WZ segments in the ZB lattice. Accordingly, the density of twin planes in these nanocrystals could influence the ratio of WZ/ZB phases.

Series of modeling on $\mathrm{Cd}_{1-x} \mathrm{Zn}_{x} \mathrm{~S}$ twinning nanocrystals were thus carried out. The calculations were firstly executed on the twin planes in pure $\mathrm{CdS}$ and $\mathrm{ZnS}$ nanocrystals, respectively. Linear transformation of band structures from CdS to $\mathrm{ZnS}$ was then applied considering the solid solution properties of $\mathrm{Cd}_{1-x} \mathrm{Zn}_{x} \mathrm{~S} \quad(0 \leq x \leq 1)$. Band structure calculations indicated type-II staggered band alignment around the twin plane, with a $\mathrm{CB}$ offset of $46 \mathrm{meV}$ and a valence band offset of $115 \mathrm{meV}$ for $\mathrm{CdS}$, as well as a CB offset of $98 \mathrm{meV}$ and a valence band offset of

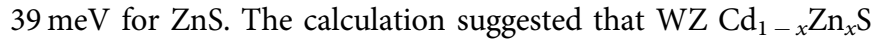
could occupy a higher $\mathrm{CB}$ and valence band (VB) position than its $\mathrm{ZB}$ counterpart. The results were further confirmed by calculations of the plane-averaged charge distribution of the $\mathrm{VB}$ maximum (VBM) and $\mathrm{CB}$ minimum (CBM) states. As shown in Fig. 4, the VBM state was more localized in the $\mathrm{WZ}$ region (near twin boundary), whereas CBM was more localized in the $\mathrm{ZB}$ region (away from twin boundary). These results are in well agreement with those reported for bulk $\mathrm{ZnS}$ and CdS semiconductors $^{36,37}$. By linear approximation, similar type-II staggered band alignment was obtained in $\mathrm{Cd}_{0.5} \mathrm{Zn}_{0.5} \mathrm{~S}$, with a WZ CB $106.5 \mathrm{meV}$ above that of $\mathrm{ZB}$ and a WZ VB $42.5 \mathrm{meV}$ above that of $\mathrm{ZB}$. The average band gap of the twinning $\mathrm{Cd}_{0.5} \mathrm{Zn}_{0.5} \mathrm{~S}$ nanorod was determined to be $2.62 \mathrm{eV}$ according to the Kubelka-Munk method from the ultraviolet-vis spectrum shown in Supplementary Fig. S5b ${ }^{38}$.

Based on above analysis, Fig. 5 gives the structural configuration (Fig. 5a) and band positions (Fig. 5b) for our twinning $\mathrm{Cd}_{0.5} \mathrm{Zn}_{0.5} \mathrm{~S}$. For comparison, $\mathrm{ZB} / \mathrm{WZ}$ segments distribution in a real $\mathrm{Cd}_{0.5} \mathrm{Zn}_{0.5} \mathrm{~S}$ nanorod twinning crystals was also provided. As shown by Fig. 5c, periodic light/dark contrast results from alternating twins along the $\langle 111\rangle$ crystalline direction of $\mathrm{Cd}_{0.5} \mathrm{Zn}_{0.5} \mathrm{~S}$ nanorod could be clearly observed. Unlike traditional heterojunctions formed by coupling two different semiconductor

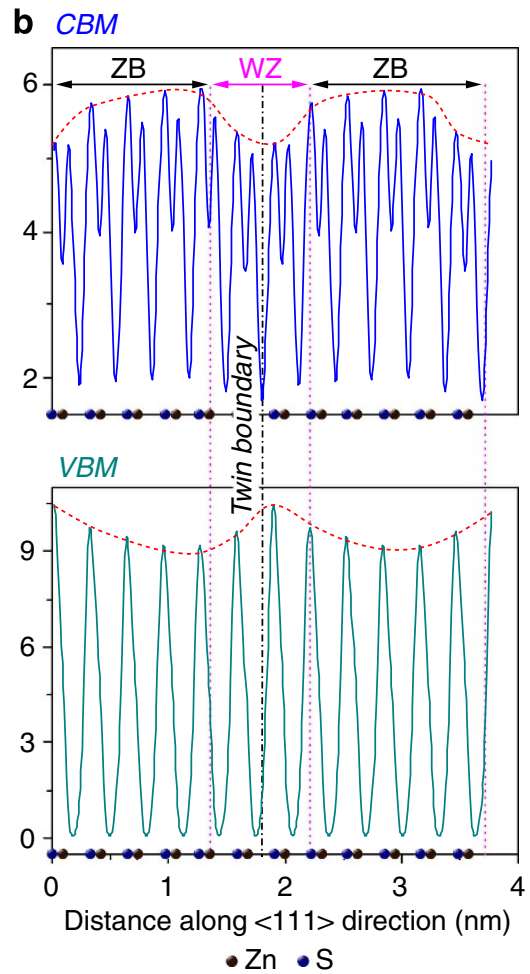

Figure 4 | Plane averaged charge densities round twin planes. (a) CB minimum (CBM) (up) and VB maximum (VBM) (down) states of zinc blende (ZB) CdS with one twin plane at the center region. (b) CBM (up) and VBM (down) states of ZB ZnS with one twin plane at the center region. 


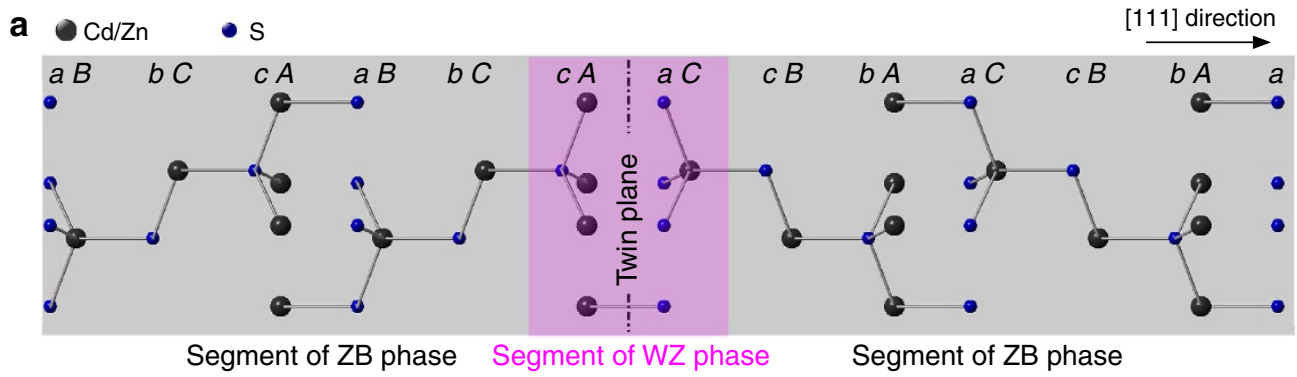

b

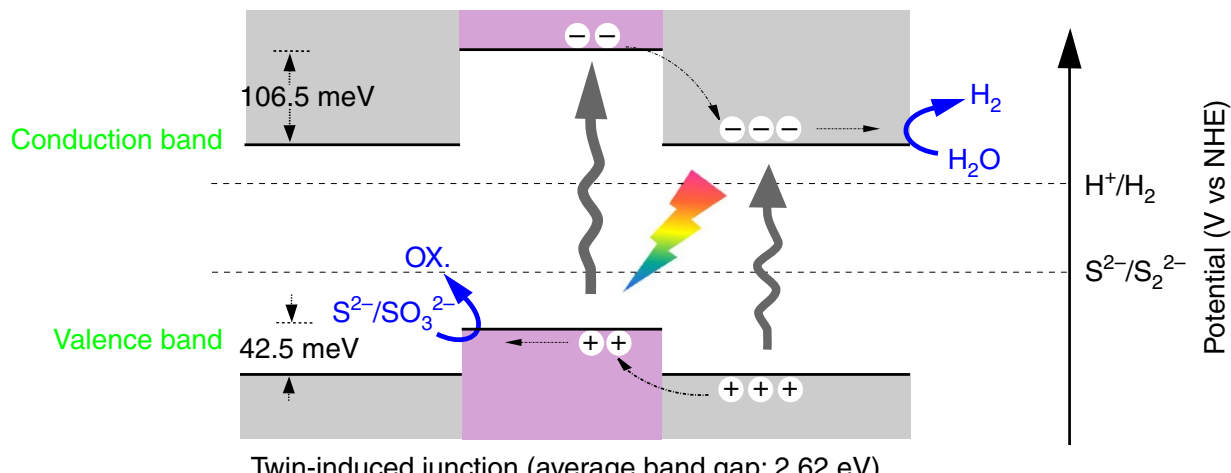

Twin-induced junction (average band gap: $2.62 \mathrm{eV}$ )

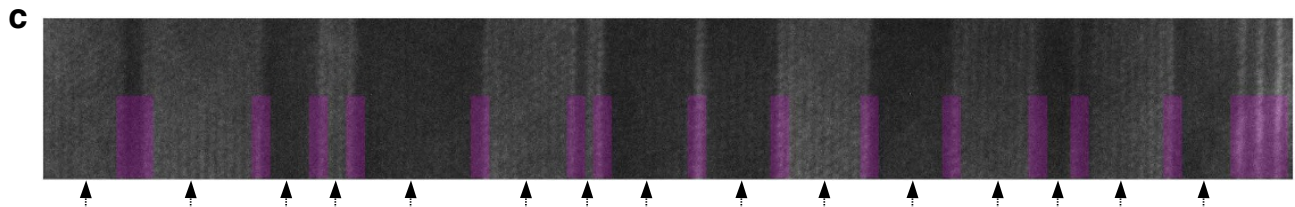

Figure 5 | Schematics of the homojunction. (a) Ball-stick structure of a typical twin boundary in a zinc blende (ZB) nanorystal to form ZB-WZ-ZB homojunction. (b) Mechanism for photo-generated charge separation over the $Z B-W Z-Z B$ homojunction with staggered band alignment in $C d_{0.5} Z n_{0.5} S$ photocatalyst. (c) A schematic illustration of the parallel homojunctions in a real $\mathrm{Cd}_{0.5} \mathrm{Zn}_{0.5} \mathrm{~S}$ nanorod (from TEM image), the black arrows and pink squares indicate the segments of ZB and WZ structures, respectively.

materials, the staggered band alignment in our $\mathrm{Cd}_{0.5} \mathrm{Zn}_{0.5} \mathrm{~S}$ was considered to act as a special homojunction, as highlighted in pink regions of Fig. 5. As illustrated in Fig. 5b, the up-shift of both $\mathrm{CB}$ and valence band energy levels for $\mathrm{WZ}$ segment compared with that of $\mathrm{ZB}$ segments could simultaneously lead to efficient separation of both photo-excited electrons and holes. This is obviously different from most of heterogeneous junctions that can only separate either electrons or holes alone ${ }^{23}$. Large distribution and the close interconnection at the atomic level of two phases (ZB and WZ) and the formation of such homojunctions are believed to favour the vectorial transfer of photoinduced charges, which has been extensively explored by heterojunction design ${ }^{23,26,28,39}$.

Formation mechanism of 1D homojunctions. Johansson et al. ${ }^{40}$ found that higher growth temperatures increased the likelihood of twin formation and in the mass-transport limited process. It is therefore considered that if hydrothermal method is to be used, relatively high hydrothermal treatment temperature is needed. This is the reason why we have chosen hydrothermal treatment temperature of as high as $230^{\circ} \mathrm{C}$.

To further investigating the underlying mechanism for the formation of homojunctions in our $\mathrm{Cd}_{0.5} \mathrm{Zn}_{0.5} \mathrm{~S}$ nanorod, we then prepared $\mathrm{Cd}_{0.5} \mathrm{Zn}_{0.5} \mathrm{~S}$ nanocrystals with similar sizes, crystal structures and light absorption properties by simply changing the coordination agent for comparison (Supplementary Fig. S7).

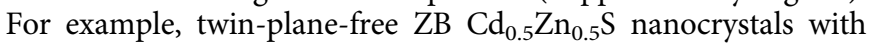

spherical polyhedral shapes (denoted as $\mathrm{Cd}_{0.5} \mathrm{Zn}_{0.5} \mathrm{~S}$-EN0) and $\mathrm{ZB}$ $\mathrm{Cd}_{0.5} \mathrm{Zn}_{0.5} \mathrm{~S}$ twinning nanorods with an aspect ratio of $c a .1 .9$ (denoted as $\mathrm{Cd}_{0.5} \mathrm{Zn}_{0.5} \mathrm{~S}$-EN5) were obtained by adding 0 and $5 \mathrm{ml}$ ethylenediamine (EN), respectively. By contrast, twinning $\mathrm{ZB} \mathrm{Cd}_{0.5} \mathrm{Zn}_{0.5} \mathrm{~S}$ nanocrystals with spherical polyhedral shapes were obtained by adding $\mathrm{NaOH}$ instead of EN (denoted as $\mathrm{Cd}_{0.5} \mathrm{Zn}_{0.5} \mathrm{~S}-\mathrm{NaOH}$; this material has been previously prepared by us using a traditional hydrothermal method ${ }^{31}$ ). Clearly, twinning structure cannot be obtained in hydrothermal reaction at $230^{\circ} \mathrm{C}$ in the absence of $\mathrm{OH}^{-}$or EN. It indicates that high temperature alone is not enough for the formation of twinning structure and the existence of $\mathrm{OH}^{-}$or $\mathrm{EN}$ is also indispensible in the hydrothermal preparation of twinning $\mathrm{Cd}_{0.5} \mathrm{Zn}_{0.5} \mathrm{~S}$. It has been reported that the difference in mass transportation for the various elements is crucial for the formation of twinning structures. Taking VLS growth of twinning GaP (gold as seed/catalyst) as an example, due to its extremely low solubility in gold, phosphorous has to migrate to the growth interface from the vapor phase to complete the $\mathrm{GaP}$ growth. In contrast, $\mathrm{Ga}$ has a quite good solubility in gold. Therefore, the growth can be greatly slowed down by the slow mass transportation of $\mathrm{P}$. In this masstransport limited process, any fluctuations, such as thermal fluctuations could lead to twin-plane nucleation, due to the low energy for the formation of coherent twin plane ${ }^{40}$. In our case, $\mathrm{OH}^{-}$or EN as coordinating agent for $\mathrm{Cd}^{2+}$ and $\mathrm{Zn}^{2+}$ ions is also believed to have similar roles. The microwave method employed may lead to more thermal fluctuations in the entire synthesis process and contribute further to the twin plane 

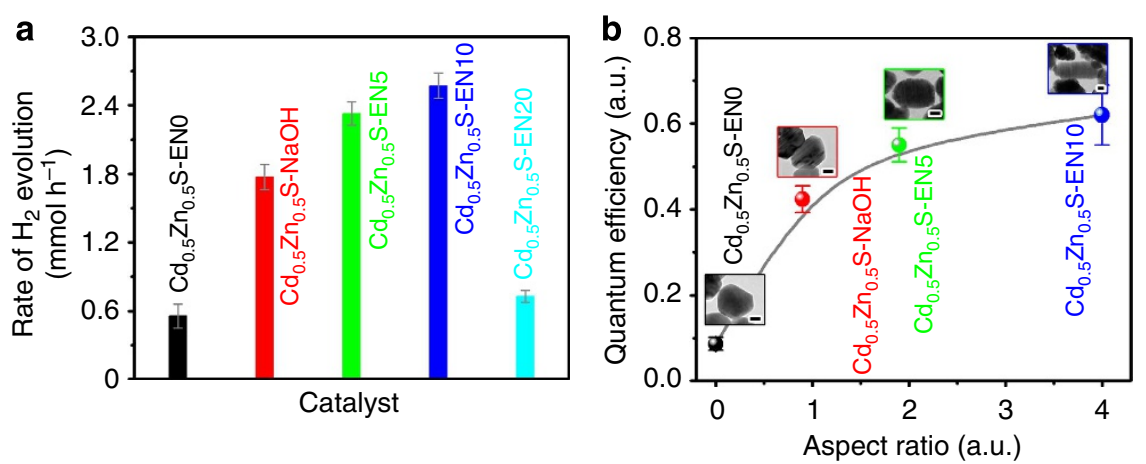

Figure 6 | Photocatalytic activity of various $\mathbf{C d}_{\mathbf{0 . 5}} \mathbf{Z n}_{\mathbf{0 . 5}} \mathbf{S}$ samples. (a) The initial hydrogen generation rate of $\mathrm{H}_{2}$ evolution over $\mathrm{Cd}_{0.5} \mathrm{Zn} \mathrm{n}_{0.5} \mathrm{~S}-\mathrm{ENO}$, $\mathrm{Cd}_{0.5} \mathrm{Zn}_{0.5} \mathrm{~S}-\mathrm{NaOH}, \mathrm{Cd}_{0.5} \mathrm{Zn}_{0.5} \mathrm{~S}-\mathrm{EN5}, \mathrm{Cd}_{0.5} \mathrm{Zn}_{0.5} \mathrm{~S}-\mathrm{EN} 10$ and $\mathrm{Cd}_{0.5} \mathrm{Zn}_{0.5} \mathrm{~S}-\mathrm{EN} 2 \mathrm{O}$ with $5 \mathrm{~h}$ tests. (b) QEs obtained over $\mathrm{Cd}_{0.5} \mathrm{Zn}_{0.5} \mathrm{~S}$ nanocrystals of twin-free spherical polyhedrons, twinning spherical polyhedrons, twinning nanorods with aspect ratio of ca. 1.9, and twinning nanorods with aspect ratio of ca. 4.0, respectively; Calculated QEs are plotted against the average aspect ratios of various $C_{0.5} Z_{0.5} S$ nanocrystals. For the twin-free spherical $C d_{0.5} Z n_{0.5} S$ nanocrystals, the aspect ratio was defined as zero. Error bars are s.e. values of three tests $(n=3)$. Scale bars for the TEM images in the sets of $\mathbf{b}$ is $20 \mathrm{~nm}$.

formation. Moreover, twin planes in the nanocrystal, especially in a nanowire, have been reported to be beneficial for the releasing of the stored elastic energy ${ }^{26}$. These could be additional benefits facilitating the spherical to nanorod morphology transition for our $\mathrm{Cd}_{0.5} \mathrm{Zn}_{0.5} \mathrm{~S}$ nanotwins.

It is also noteworthy that EN has played a crucial role for in the formation our 1D-nanorod structure. In fact, similar role of EN has also been found for the formation of many anisotropic structures on various materials, such as nanowire, nanorod, and nanosheet/plate and so on ${ }^{41-45}$. It was proposed that atoms on coherent $\{111\}$ twin plane possessed high coordination number and had relatively low formation energy even comparable to the normal $\{111\}$ facets ${ }^{27,46-48}$. The [111] preferential growth of our twinning $\mathrm{Cd}_{0.5} \mathrm{Zn}_{0.5} \mathrm{~S}$ nanorod is therefore believed to be also due to the capping effect of EN.

Comparative validation on the role of $1 \mathrm{D}$ homojunctions. Photocatalytic tests over these various $\mathrm{Cd}_{0.5} \mathrm{Zn}_{0.5} \mathrm{~S}$ samples were then carried out to evaluate their photocatalytic performances. As shown in Fig. 6a, samples $\mathrm{Cd}_{0.5} \mathrm{Zn}_{0.5} \mathrm{~S}-\mathrm{NaOH}, \mathrm{Cd}_{0.5} \mathrm{Zn}_{0.5} \mathrm{~S}-\mathrm{EN} 5$ and $\mathrm{Cd}_{0.5} \mathrm{Zn}_{0.5} \mathrm{~S}$-EN10 exhibited much higher activity than that without twinning structure. This again demonstrated the crucial role of twin-induced junctions in enhancing photocatalytic activity. QEs at $425 \mathrm{~nm}$ for the four $\mathrm{Cd}_{0.5} \mathrm{Zn}_{0.5} \mathrm{~S}$ photocatalysts were then evaluated and plotted against the aspect ratio of the various prepared $\mathrm{Cd}_{0.5} \mathrm{Zn}_{0.5} \mathrm{~S}$ nanocrystals. As shown in Fig. $6 \mathrm{~b}$, with similar morphology and size, the $\mathrm{QE}$ of twinning $\mathrm{Cd}_{0.5} \mathrm{Zn}_{0.5} \mathrm{~S}$ spherical polyhedrons ( $c a$. 40\%) were found to be about 4.4 times that without nano-twins (ca. 9\%). The efficiency can be further improved to $55 \%$ over the twinning nanorods with an aspect ratio of 1.9. The highest QE of $62 \%$ as aforementioned was obtained over nanorods with an aspect ratio of $\sim 4.0$. It is noteworthy that the highest QE reported so far for photocatalytic hydrogen production without noble metal loading is $51 \%$ (ref. 16). While our photocatalyst reaches $62 \%$. Clearly, the enhanced charge separation and consequent high activity has a close relationship with the large aspect ratios of the twinning nanocrystal.

Time-resolved photoluminescence emission decay spectra, which have been widely used to evaluate the lifetime of photogenerated carriers in semiconducting materials, were then recorded for various $\mathrm{Cd}_{0.5} \mathrm{Zn}_{0.5} \mathrm{~S}$ samples. All the samples were excited at $305 \mathrm{~nm}$ and the data were collected at $495 \mathrm{~nm}$, which corresponds to the emission peak of $\mathrm{Cd}_{0.5} \mathrm{Zn}_{0.5} \mathrm{~S}$. The lifetime $(\tau)$ was calculated by an exponential decay approach according to the equation $I=I_{0} e^{-t / \tau}+b$, where $I$ is the impulse function decay at time $t$. As shown in Supplementary Fig. S8, the excited carrier in $\mathrm{Cd}_{0.5} \mathrm{Zn}_{0.5} \mathrm{~S}$-EN0 presented the shortest lifetime $(\tau=2.36 \pm 0.12$ $\mathrm{ns})$. By contrast, the emission decay times for those $\mathrm{Cd}_{0.5} \mathrm{Zn}_{0.5} \mathrm{~S}$ samples with nano-twins were obviously slowed down. $\tau$ is $3.25 \pm 0.12 \mathrm{~ns}$ for spherical nano-twins and it is $4.02 \pm 0.25 \mathrm{~ns}$ and $5.41 \pm 0.41 \mathrm{~ns}$ for twinning $\mathrm{Cd}_{0.5} \mathrm{Zn}_{0.5} \mathrm{~S}$ with aspect ratios of ca. 1.9 and ca. 4.0, respectively. Obviously, twinning nanocrystals with larger aspect ratios will lead to prolonged lifetime of photogenerated charges.

More importantly, by plotting QEs as a function of the aspect ratio of TSL in these $\mathrm{Cd}_{0.5} \mathrm{Zn}_{0.5} \mathrm{~S}$ nanocrystals and taking the aspect ratio of non-twin polyhedral $\mathrm{Cd}_{0.5} \mathrm{Zn}_{0.5} \mathrm{~S}$-EN0 as zero (spherical $\mathrm{Cd}_{0.5} \mathrm{Zn}_{0.5} \mathrm{~S}$ without twin planes), a well-defined upward curve was generated. Figure $6 \mathrm{~b}$ indicates that a higher aspect ratio of twin photocatalyst, such as nanowire, is expected to result in further improved efficiency. This finding again demonstrated the importance of long-range ordered TSL for the enhancement of photocatalytic activity, which cannot be obtained in traditional spherical structures as we have previously reported for twinning $\mathrm{Cd}_{0.5} \mathrm{Zn}_{0.5} \mathrm{~S}$ nanoparticles ${ }^{31}$.

\section{Discussion}

The activity of photocatalytic process has long relied on the crystal structure of semiconductors to facilitate charge separation. As aforementioned, the unique twin induced TSL with a long-range order has been obtained by our EN-assisted hightemperature hydrothermal preparation. The resultant homojunctions could lead to significantly enhanced photocatalytic activity. Both our theoretical and experimental investigations have provided direct proof of the crucial role of twin-induced homojunction in prompting charge separation and photocatalytic reactions. As shown in Fig. 5b, the photogenerated electrons and holes can be spatially separated to the ZB (away from the twin plane) and WZ (to twin plane) regions, respectively, because of the presence of this homojunction.

On the other hand, semiconductors with one dimensional (1D) nanostructures (corresponding large aspect ratios) have been proposed to have higher charge-separation ability than their bulk counterpart ${ }^{49-51}$. For our $\mathrm{Cd}_{0.5} \mathrm{Zn}_{0.5} \mathrm{~S}$ twinning nanorods, ordered long-range twinning planes with preferred orientation of [111] direction were achieved. TSL is expected to be formed from twinning planes of long-range order, which leads to the formation of series homojunctions with short spacing. In this case, both excited electrons and holes can be efficiently separated in shorter travelling distance before they undergo recombination. Such 
densely distributed homojunctions are obviously advantageous than the single buried-in homojunction as reported by Lin et al. ${ }^{52}$, which introduced a thin p-type layer on n-type $\alpha-\mathrm{Fe}_{2} \mathrm{O}_{3}$ and created a built-in field.

In conclusion, we demonstrated the efficient charge separation and therefore very high hydrogen production without noble metal loading over our twinning $\mathrm{Cd}_{0.5} \mathrm{Zn}_{0.5} \mathrm{~S}$ nanorod photocatalysts. The key to this achievement is the successful formation of highdensity (111)/[11-2]-type TSL in certain dimension. Long-range ordered twinning planes with preferred [111] orientation leads to near periodical and dense distribution of a series of homojunctions. These homojunctions have been demonstrated to be very efficient in separating photo-generated charges and contributed to prolonged lifetime of both photo-generated electrons and holes. It is predicted that even higher photocatalytic activity could be achieved if larger aspect ratios, that is, longer $\mathrm{Cd}_{0.5} \mathrm{Zn}_{0.5} \mathrm{~S}$ twinned nanorods, could be obtained. The ability to form such a homojunction by twin planes in a semiconductor presents a promising strategy for precise band engineering without introducing foreign materials or elements as required by traditional heterojunctions and provides new insight into the fabrication of novel structures for photocatalytic and photoelectrochemical applications.

\section{Methods}

Syntheses. $\mathrm{Cd}_{0.5} \mathrm{Zn}_{0.5} \mathrm{~S}$-EN10 nanocrystals were prepared by sequentially adding $10 \mathrm{ml}$ of EN and $25 \mathrm{mmol}$ thioacetamide of to a $40-\mathrm{ml}$ aqueous solution containing $10 \mathrm{mmol}$ of cadmium acetate $\left(\mathrm{Cd}\left(\mathrm{CH}_{3} \mathrm{COO}\right)_{2}\right)$ and $10 \mathrm{mmol}$ of zinc acetate $\left(\mathrm{Zn}\left(\mathrm{CH}_{3} \mathrm{COO}\right)_{2}\right)$. The total volume of the reaction was adjusted to be $60 \mathrm{ml}$ by introducing additional water. The entire process was carried out under magnetic stirring. The reaction mixture was further stirred for $20 \mathrm{~min}$, sealed in a $100 \mathrm{ml}-$ capacity Teflon-lined autoclave, and heated to $230^{\circ} \mathrm{C}$ at a ramping speed of $30^{\circ} \mathrm{C} \mathrm{min}^{-1}$ in the presence of microwave irradiation. The reaction was allowed to proceed for $30 \mathrm{~min}$. After cooling, the product was separated by centrifugation, washed several times with deionized water and ethanol, and dried at $80^{\circ} \mathrm{C}$ for $5 \mathrm{~h}$ in a vacuum oven. Preparation of other $\mathrm{Cd}_{1-x} \mathrm{Zn}_{x} \mathrm{~S}$-EN10 nanocrystals is the same with the synthesis of $\mathrm{Cd}_{0.5} \mathrm{Zn}_{0.5} \mathrm{~S}$-EN10 nanocrystal except employing a different molar ratio of $\mathrm{Cd}\left(\mathrm{CH}_{3} \mathrm{COO}\right)_{2} / \mathrm{Zn}\left(\mathrm{CH}_{3} \mathrm{COO}\right)_{2}$. For the synthesis of other $\mathrm{Cd}_{0.5} \mathrm{Zn}_{0.5} \mathrm{~S}$ photocatalysts for comparison, the processes were similar to that of $\mathrm{Cd}_{0.5} \mathrm{Zn}_{0.5} \mathrm{~S}$-EN10 synthesis, except that 5 and $20 \mathrm{ml}$ of EN, and $10 \mathrm{ml}$ of sodium hydroxide $(\mathrm{NaOH}, 4 \mathrm{M})$ were used (instead of $10 \mathrm{ml} \mathrm{EN}$ ). The total volume of the reaction was also adjusted to be $60 \mathrm{ml}$ by introducing additional water.

Photocatalytic reactions. Photocatalytic reactions of hydrogen production from water were conducted in a gas-closed system with a side irradiation Pyrex cell (using PLS-SXE300/300UV Xe lamp irradiation) at $35^{\circ} \mathrm{C}$. The photocatalyst powder $(0.1 \mathrm{~g})$ was dispersed with a magnetic stirrer in an aqueous solution $(180 \mathrm{ml})$ containing sodium sulphide $\left(\mathrm{Na}_{2} \mathrm{~S}, 0.35 \mathrm{M}\right)$ and sodium sulphite $\left(\mathrm{Na}_{2} \mathrm{SO}_{3}\right.$, $0.25 \mathrm{M})$ as electron donors. The photocatalysts were irradiated with visible light $(\lambda \geq 430 \mathrm{~nm})$ through a cutoff filter from the Xe lamp. The intensity and number of photons of the two light sources were measured with a fiber optic spectrometer. The amount of $\mathrm{H}_{2}$ gas was determined by online thermal conductivity detector gas chromatography ( $\mathrm{NaX}$ zeolite column, thermal conductivity detector detector, and $\mathrm{N}_{2}$ carrier) or drainage. QEs were measured using $425 \mathrm{~nm}$ band pass filters and an irradiation meter and were defined by equation (1):

$$
\mathrm{QE}=2 N_{\mathrm{H}} / N_{\mathrm{P}}
$$

where $N_{\mathrm{H}}$ is the number of evolved $\mathrm{H}_{2}$ molecules and $N_{\mathrm{P}}$ is the number of incident photons.

No $\mathrm{H}_{2}$ evolution was detected under blank experiments carried out in the absence of catalysts or /and visible light irradiation. The catalytic feature of $\mathrm{Cd}_{0.5} \mathrm{Zn}_{0.5} \mathrm{~S}$ twinning rods was further certified by their unchanged structural, optical and morphologic properties, recovered and characterized after 20-h hydrogen production (see Supplementary Fig. S9).

Modelling details. The rotational twin boundary was modelled by a $12 \mathrm{H}$ supercell, as shown in Fig. 5a and Supplementary Fig. S3. Two equivalent boundaries were actually included in the supercell with each boundary well separated. Charge densities at the VBM and CBM and a potential barrier across the twin boundary were calculated using the Vienna ab initio simulation package ${ }^{53,54}$. Generalized gradient approximation ${ }^{55}$ within density functional theory and the projector augmented wave potentials ${ }^{56}$ were used ${ }^{55,56}$. A kinetic energy cutoff of $380 \mathrm{eV}$ was chosen, and a $9 \times 9 \times 1$ Monkhorst-Park k-point mesh was used to sample the hexagonal two-dimensional Brillouin zone ${ }^{57}$. The twin boundary was fully relaxed until the force acting on ions was smaller than $0.01 \mathrm{eV} \AA^{-1}$. The band decomposed charge density was extracted to depict charge distribution along the direction normal to the boundary. The macro-averaged total potential was used to determine the potential barrier across the boundary. The ZB/WZ interface was modeled by a $(3 \mathrm{C})_{2}(2 \mathrm{H})_{3}$ supercell. The band offset across the $\mathrm{ZB} / \mathrm{WZ}$ interface was obtained using macro-averaged total potential by combining the interface and individual $\mathrm{ZB}$ and WZ bulk calculations ${ }^{58}$.

Characterizations. The X-ray diffraction patterns of the prepared photocatalysts were confirmed by an X'Pert PRO diffractometer using Cu Ka $(\lambda=0.1538 \mathrm{~nm})$ radiation with a constant instrument parameter. All samples were scanned between $10^{\circ}$ and $90^{\circ}$ with a step size of $0.033^{\circ}$. Diffuse reflectance ultraviolet-vis spectra were obtained on a Hitachi U-4100 spectrometer equipped with a laboratorysphere diffuse reflectance accessory. The crystallite morphologic micrographs were observed by JEOL JSM-6700F field emission SEM). TEM JEM-3010 and transmission electron microscope FEI Tecnai F30. STEM and EDX analyses were performed using an ARM200 microscope with a STEM Cs corrector (JEOL, Tokyo, Japan). The room temperature photoluminescent emission spectra and timeresolved photoluminescent emission decay spectra were measured on a PTI QM-4 fluorescence spectrophotometer.

\section{References}

1. Fujishima, A. \& Honda, K. Electrochemical photolysis of water at a semiconductor electrode. Nature 238, 37-38 (1972).

2. Bard, A. J. \& Fox, M. A. Artificial photosynthesis: solar splitting of water to hydrogen and oxygen. Acc. Chem. Res. 28, 141-145 (1995)

3. Kudo, A. \& Miseki, Y. Heterogeneous photocatalyst materials for water splitting. Chem. Soc. Rev. 38, 253-278 (2009).

4. Tachibana, Y., Vayssieres, L. \& Durrant, J. R. Artificial photosynthesis for solar water-splitting. Nat. Photon. 6, 511-518 (2012).

5. Li, Z., Luo, W., Zhang, M., Feng, J. \& Zou, Z. Photoelectrochemical cells for solar hydrogen production: current state of promising photoelectrodes, methods to improve their properties, and outlook. Energy Environ. Sci. 6, 347-370 (2013).

6. Chen, X., Shen, S., Guo, L. \& Mao, S. S. Semiconductor-based photocatalytic hydrogen generation. Chem. Rev. 110, 6503-6570 (2010).

7. Reber, J. \& Rusek, M. Photochemical hydrogen production with platinized suspensions of cadmium sulfide and cadmium zinc sulfide modified by silver sulfide. J. Phys. Chem. 90, 824-834 (1986).

8. Zou, Z., Ye, J., Sayama, K. \& Arakawa, H. Direct splitting of water under visible light irradiation with an oxide semiconductor photocatalyst. Nature $\mathbf{4 1 4}$ 625-627 (2001).

9. Maeda, K. et al. Photocatalyst releasing hydrogen from water. Nature 440, 295-295 (2006).

10. Xu, X., Randorn, C., Efstathiou, P. \& Irvine, J. T. S. A red metallic oxide photocatalyst. Nat. Mater. 11, 595-598 (2012).

11. Kato, H., Asakura, K. \& Kudo, A. Highly efficient water splitting into $\mathrm{H}_{2}$ and $\mathrm{O}_{2}$ over lanthanum-doped $\mathrm{NaTaO}_{3}$ photocatalysts with high crystallinity and surface nanostructure. J. Am. Chem. Soc. 125, 3082-3089 (2003).

12. Wang, X. et al. A metal-free polymeric photocatalyst for hydrogen production from water under visible light. Nat. Mater. 8, 76-80 (2009).

13. Chen, X., Liu, L., Yu, P. Y. \& Mao, S. S. Increasing solar absorption for photocatalysis with black hydrogenated titanium dioxide nanocrystals. Science 331, 746-750 (2011)

14. Mukherji, A. et al. $\mathrm{N}$-doped $\mathrm{CsTaWO}_{6}$ as a new photocatalyst for hydrogen production from water splitting under solar irradiation. Adv. Funct. Mater. 21, 126-132 (2011).

15. Maeda, K. et al. GaN:ZnO solid solution as a photocatalyst for visible-lightdriven overall water splitting. J. Am. Chem. Soc. 127, 8286-8287 (2005).

16. Zhang, W., Wang, Y., Wang, Z., Zhong, Z. \& Xu, R. Highly efficient and noble metal-free NiS/CdS photocatalysts for $\mathrm{H}_{2}$ evolution from lactic acid sacrificial solution under visible light. Chem. Commun. 46, 7631-7633 (2010).

17. Liu, M., Du, Y., Ma, L., Jing, D. \& Guo, L. Manganese doped cadmium sulfide nanocrystal for hydrogen production from water under visible light. Int. J. Hydrogen Energy 37, 730-736 (2012).

18. Tada, H., Mitsui, T., Kiyonaga, T., Akita, T. \& Tanaka, K. All-solid-state $\mathrm{Z}$-scheme in CdS-Au-TiO 2 three-component nanojunction system. Nat. Mater. 5, 782-786 (2006).

19. Iwase, A., Ng, Y. H., Ishiguro, Y., Kudo, A. \& Amal, R. Reduced graphene oxide as a solid-state electron mediator in Z-scheme photocatalytic water splitting under visible light. J. Am. Chem. Soc. 133, 11054-11057 (2011).

20. Yan, H. et al. Visible-light-driven hydrogen production with extremely high quantum efficiency on Pt-PdS/CdS photocatalyst. J. Catal. 266, 165-168 (2009).

21. Mayer, M. T., Lin, Y., Yuan, G. \& Wang, D. Forming heterojunctions at the nanoscale for improved photoelectrochemical water splitting by semiconductor materials: case studies on hematite. Acc. Chem. Res. 46, 1558-1566 (2013). 
22. Wang, X. et al. Photocatalytic overall water splitting promoted by an $\alpha-\beta$ phase junction on $\mathrm{Ga}_{2} \mathrm{O}_{3}$. Angew. Chem. Int. Ed. 51, 13089-13092 (2012).

23. Zong, X. et al. Enhancement of photocatalytic $\mathrm{H}_{2}$ evolution on CdS by loading $\mathrm{MoS}_{2}$ as cocatalyst under visible light irradiation. J. Am. Chem. Soc. 130, 7176-7177 (2008).

24. Algra, R. E. et al. Twinning superlattices in indium phosphide nanowires. Nature 456, 369-372 (2008).

25. Akiyama, T., Yamashita, T., Nakamura, K. \& Ito, T. Band alignment tuning in twin-plane superlattices of semiconductor nanowires. Nano. Lett. 10, 4614-4618 (2010).

26. Li, Q. et al. Size-dependent periodically twinned ZnSe nanowires. Adv. Mater. 16, 1436-1440 (2004).

27. Caroff, P. et al. Controlled polytypic and twin-plane superlattices in iii-v nanowires. Nat. Nanotech. 4, 50-55 (2009).

28. Yan, Y., Noufi, R. \& Al-Jassim, M. M. Grain-boundary physics in polycrystalline CuInSe2 revisited: experiment and theory. Phys. Rev. Lett. 96, 205501 (2006)

29. Verheijen, M. A., Immink, G., de Smet, T., Borgström, M. T. \& Bakkers, E. P. A. M. Growth kinetics of heterostructured GaP-GaAs nanowires. J. Am. Chem. Soc. 128, 1353-1359 (2006).

30. Xiong, Q., Wang, J. \& Eklund, P. C. Coherent twinning phenomena: towards twinning superlattices in III $-\mathrm{V}$ semiconducting nanowires. Nano. Lett. 6, 2736-2742 (2006).

31. Liu, M., Wang, L., Lu, G., Yao, X. \& Guo, L. Twins in $\mathrm{Cd}_{1-x} \mathrm{Zn}_{x} \mathrm{~S}$ solid solution: highly efficient photocatalyst for hydrogen generation from water. Energy Environ. Sci. 4, 1372-1378 (2011)

32. Chen, M. et al. Deformation twinning in nanocrystalline aluminum. Science 300, 1275-1277 (2003).

33. Lu, L., Shen, Y., Chen, X., Qian, L. \& Lu, K. Ultrahigh strength and high electrical conductivity in copper. Science 304, 422-426 (2004).

34. Priya, R. \& Kanmani, S. Batch slurry photocatalytic reactors for the generation of hydrogen from sulfide and sulfite waste streams under solar irradiation. Solar Energy 83, 1802-1805 (2009).

35. Yang, Y. et al. Twinned $\mathrm{Zn}_{2} \mathrm{TiO}_{4}$ spinel nanowires using $\mathrm{ZnO}$ nanowires as a template. Adv. Mater. 19, 1839-1844 (2007).

36. Wei, S.-H. \& Zhang, S. B. Structure stability and carrier localization in CdX $(\mathrm{X}=\mathrm{S}, \mathrm{Se}, \mathrm{Te})$ semiconductors. Phys. Rev. B 62, 6944-6947 (2000).

37. Murayama, M. \& Nakayama, T. Chemical trend of band offsets at wurtzite/ zinc-blende heterocrystalline semiconductor interfaces. Phys. Rev. B 49, 4710-4724 (1994).

38. Kim, II Y., Atherton, S., Brigham, E. S. \& Mallouk, T. E. Sensitized layered metal oxide semiconductor particles for photochemical hydrogen evolution from nonsacrificial electron donors. J. Phys. Chem. 97, 11802-11810 (1993).

39. Maeda, K. et al. Photocatalytic Overall water splitting promoted by two different cocatalysts for hydrogen and oxygen evolution under visible light. Angew. Chem. Int. Ed. 49, 4096-4099 (2010).

40. Johansson, J. et al. Structural properties of $<111>$ B-oriented III-V nanowires. Nat. Mater. 5, 574-580 (2006).

41. Tong, H. et al. Lead chalcogenide nanotubes synthesized by biomoleculeassisted self-assembly of nanocrystals at room temperature. Angew. Chem. 118, 7903-7906 (2006)

42. Mi, L. et al. One-pot synthesis and the electrochemical properties of nanostructured nickel selenide materials with hierarchical structure. Cryst. Eng. Comm. 15, 2624-2630 (2013)

43. $\mathrm{Hu}, \mathrm{Q}$. et al. Selective preparation and enhanced microwave electromagnetic characteristics of polymorphous $\mathrm{ZnO}$ architectures made from a facile one-step ethanediamine-assisted hydrothermal approach. Cryst. Eng. Comm. 15, 1314-1323 (2013)

44. Jin, R., Chen, G., Wang, Q., Sun, J. \& Wang, Y. A facile solvothermal synthesis of hierarchical $\mathrm{Sb}_{2} \mathrm{Se}_{3}$ nanostructures with high electrochemical hydrogen storage ability. J. Mater. Chem. 21, 6628-6635 (2011).
45. Jin, R., Chen, G., Pei, J., Xua, H. \& Lv, Z. S. Solvothermal synthesis and growth mechanism of ultrathin $\mathrm{Sb}_{2} \mathrm{Te}_{3}$ hexagonal nanoplates with thermoelectric transport properties. RSC Adv. 2, 1450-1456 (2012).

46. Glas, F., Harmand, J.-C. \& Patriarche, G. Why does wurtzite form in nanowires of III-V zinc blende semiconductors? Phys. Rev. Lett. 99, 146101 (2007).

47. Hurlea, D. T. J. \& Rudolph, P. A brief history of defect formation, segregation, faceting, and twinning in melt-grown semiconductors. J. Crystal Growth 264, 550-564 (2004).

48. Korgel, B. A. Semiconductor nanowires: Twins cause kinks. Nat. Mater. 5, 521-522 (2006).

49. Wang, Z. L. \& Song, J. Piezoelectric nanogenerators based on zinc oxide nanowire arrays. Science 312, 242-246 (2006).

50. Jennings, J. R., Ghicov, A., Peter, L. M., Schmuki, P. \& Walker, A. B. Dyesensitized solar cells based on oriented $\mathrm{TiO}_{2}$ nanotube arrays: transport, trapping, and transfer of electrons. J. Am. Chem. Soc. 130, 13364-13372 (2008)

51. Wu, K., Zhu, H., Liu, Z., Rodríguez-Córdoba, W. \& Lian, T. Ultrafast charge separation and long-lived charge separated state in photocatalytic CdS-Pt nanorod heterostructures. J. Am. Chem. Soc. 134, 10337-10340 (2012).

52. Lin, Y. J. et al. Growth of p-type hematite by atomic layer deposition and its utilization for improved solar water splitting. J. Am. Chem. Soc. 134, 5508-5511 (2012).

53. Kresse, G. \& Furthmuller, J. Efficient iterative schemes for ab initio total-energy calculations using a plane-wave basis set. Phys. Rev. B 54, 11169-11186 (1996)

54. Kresse, G. \& Furthmuller, J. Efficiency of ab-initio total energy calculations for metals and semiconductors using a plane-wave basis set. Comput. Mater. Sci. 6 15-50 (1996)

55. Perdew, J. P., Burke, K. \& Ernzerhof, M. Generalized gradient approximation made simple. Phys. Rev. Lett. 77, 3865-3868 (1996).

56. Kresse, G. \& Joubert, D. From ultrasoft pseudopotentials to the projector augmented-wave method. Phys. Rev. B 59, 1758-1775 (1999).

57. Monkhorst, H. J. \& Pack, J. D. Special points for Brillouin-zone integrations. Phys. Rev. B 13, 5188 (1976).

58. Qteish, A., Heine, V. \& Needs, R. J. Polarization, band lineups, and stability of SiC polytypes. Phys. Rev. B 45, 6534-6542 (1992).

\section{Acknowledgements}

This work was supported by the National Natural Science Foundation of China (No. 51236007, No. 51121092 and No. 21276206) and the National Basic Research Program of China (No. 2009CB220000). We also appreciate the help of Dr Lu for conducting the (S)TEM and EDX measurements at International Center for Direlectric Research (ICDR) in Xi'an Jiaotong University, and thank Y. Du and P. Guo for assistance and Dr L. Vayssieres and Dr H. Yang for helpful discussions and critical reading of the manuscript.

\section{Author contributions}

L.G., M.L. and D.J. conceived and designed the experiments. L.G. supervised the project. M.L. and D.J. performed experiments and analyzed the data. Z.Z. conducted the theoretical computations. M.L., D.J. and L.G. prepared and revised the manuscript. All authors discussed the results and commented on the manuscript.

\section{Additional information}

Supplementary Information accompanies this paper at http://www.nature.com/ naturecommunications

Competing financial interests: The authors declare no competing financial interests.

Reprints and permission information is available online at http://npg.nature.com/ reprintsandpermissions/

How to cite this article: Liu, M. et al. Twin-induced one-dimensional homojunctions yield high quantum efficiency for solar hydrogen generation. Nat. Commun. 4:2278 doi: $10.1038 /$ ncomms3278 (2013) 